\title{
Probabilidad de Deserción Estudiantil en Cursos de Matemáticas Básicas en Programas Profesionales de la Universidad de Los Andes-Venezuela
}

\author{
Samaria V. Muñoz-Camacho ${ }^{\left(1,2^{*}\right)}$, Teresita Gallardo( ${ }^{(1)}$, Meridalba Muñoz-Bravo(2) y Carlos A. Muñoz-Bravo(2) \\ (1) Escuela Politécnica Nacional, Ladrón de Guevara E11-253, Quito 170517, Ecuador \\ (2) Universidad de Los Andes, Av. Principal de Los Chorros de Milla, Mérida 5101, Mérida, Venezuela \\ (e-mail: samaria.munoz@epn.edu.ec; teresita.gallardo@epn.edu.ec; meridalb@ula.ve; cmunoz@ula.ve)
}

* Autor a quien debe ser dirigida la correspondencia

Recibido Nov. 22, 2017; Aceptado Feb. 5, 2018; Versión final Mar. 29, 2018, Publicado Ago. 2018

\begin{abstract}
Resumen
El objetivo del presente trabajo es describir las probabilidades de deserción estudiantil en cursos de matemáticas básicas para diferentes programas profesionales de la Universidad de Los Andes-Venezuela. Este trabajo es el resultado de una investigación realizada en el período de 2011 a 2016, que asumió un diseño de investigación cuantitativo y siguió la alternativa metodológica del estudio de caso único. Se aplicaron diferentes métodos del nivel teórico, empírico y estadístico-matemático. Para tal efecto, se construyó un modelo Logit que permitiera evaluar si la probabilidad de deserción puede ser predicha considerando características personales (como género) y aspectos institucionales, como el tipo de periodo académico, el tipo de curso académico y el número de estudiantes por curso. Los resultados obtenidos evidencian de manera relevante, la mayor probabilidad de desertar de un periodo académico regular, libre escolaridad, cursos de mayor de cincuenta estudiantes y género femenino.
\end{abstract}

Palabras clave: deserción estudiantil; programas profesionales; matemáticas básicas; modelos logit.

\section{Probability of Student Dropout in Courses of Basic Mathematics in Professional Programs of the Universidad de Los Andes-Venezuela}

\begin{abstract}
The objective of the present work is to describe the probability of student desertion in courses of basic mathematics for different professional programs of the University of Los Andes-Venezuela. This is the result of an investigation conducted in the period 2011 to 2016, which assumed a quantitative research design and followed the methodological alternative of a single case study. Different methods were applied at theoretical, empirical and statistical-mathematical levels. For this purpose, a Logit model was constructed to assess whether the probability of dropping out is predicted by considering personal characteristics (such as gender) and institutional aspects such as the type of academic period, the type of academic course and the number of students per course. The results show in a relevant way, the greater probability of dropping out from courses taken during a regular academic period, those having free schooling, from courses of more than fifty students and belonging to the female gender.
\end{abstract}

Keywords: student dropout; professional programs; basic math; probability; logit models 


\section{INTRODUCCIÓN}

En la actualidad, el abandono estudiantil en la Educación Superior constituye un fenómeno extendido que alcanza gran relevancia en América Latina; sin embargo, la complejidad de este fenómeno ha sido una de las causantes para su no solución. Los estudios realizados sobre la deserción estudiantil muestran un ámbito diverso y complejo, donde se estudian diferentes variables o factores desde la mirada de distintas disciplinas y modelos de análisis. Narváez (2001) señala que estos se concentran en dos grupos principales: los centrados en el docente y sus actuaciones de enseñanza y aquellos referidos a aspectos de los estudiantes. No obstante, existen otros estudios que han considerado factores asociados como los realizados por Miranda y Guzmán (2017), quienes encontraron que las razones socioeconómicas y el puntaje de ingreso a la universidad explican la deserción estudiantil en una universidad de Chile, la cual estudiaron usando la técnica de la minería datos.

Se requiere entonces un acercamiento desde lo conceptual a la categoría deserción. Al respecto, se ha considerado la afirmación de Tinto (1989, citado por Muñoz-Camacho et al. 2016) sobre el hecho de que ninguna definición puede captar en su totalidad la complejidad de este fenómeno, por lo que los investigadores deben elegir la definición que mejor se ajuste a sus objetivos y al problema a investigar. Para esta investigación se ha considerado conveniente asumir la definición de Himmel (2002), quien define la deserción como el abandono prematuro de un programa de estudios antes de alcanzar el título o grado y considera un tiempo suficientemente largo para descartar la posibilidad de que el estudiante se reincorpore; en cuanto al alcance de la deserción.

Por su parte, Hammond (2007) reseña los principales factores de riesgo asociados al abandono escolar, establecidos por el National Dropout Prevention Center de EE. UU, los cuales resultan significativos para los fines de esta investigación, al considerar entre los factores predictivos de la deserción, el dominio personal con tres dimensiones y sus indicadores. La primera dimensión es rendimiento escolar con indicadores como: bajos logros y retención sobre la edad; la segunda dimensión compromiso con la escuela con los indicadores: baja asistencia, bajas expectativas educativas, falta de esfuerzo, poca identificación con la escuela y no participación en actividades extracurriculares; por último, la tercera dimensión comportamiento escolar: mal comportamiento y agresión temprana. Se ha incluido la dimensión rendimiento escolar con el indicador bajos logros, vistos desde una orientación predictiva de la retención o la deserción. Pero, además, se han considerado otros indicadores relacionados con el rendimiento, tales como: de carácter personal: género del estudiante y de carácter institucional: tipo de periodo académico, tipo de curso académico, número de estudiantes.

Para ello se ha tenido en cuenta la prospectiva, siguiendo a "Gaston (1964), como la ciencia que estudia el futuro para comprenderlo y poder influir en él" (Mera, 2014). De igual manera se considera, como señala Mera (2014), que el pensamiento prospectivo es igual a la anticipación, ya que se realizan análisis sistémicos para integrar contextos, contenidos y procesos que conecten las variables y los diferentes actores con sus múltiples interrelaciones, en busca de discriminar con certeza dentro de grandes volúmenes de información, con el propósito de extraer, mediante procesamientos adecuados, aquellas informaciones principales, pertinentes y útiles que permitan realizar una apropiación y contextualización de la información para dar mejores respuestas dentro de los procesos de toma de decisiones.

Ortega (2008) señala que los métodos de investigación orientados a la prospectiva se pueden agrupar en tres tipos fundamentales: métodos de expertos basado en las opiniones de conocedores del problema a analizar; métodos extrapolativos basados en datos históricos que se pueden extrapolar al futuro y métodos de correlación basados en la identificación de factores relevantes y su evolución hacia el futuro. En la presente investigación se ha utilizado el tercer tipo. Se asume el análisis predictivo por ser una forma de análisis avanzado que utiliza datos nuevos e históricos para predecir la actividad futura, el comportamiento y las tendencias. Para lograrlo, es necesario la aplicación de técnicas de análisis estadístico, consultas analíticas y algoritmos automáticos de aprendizaje automático a conjuntos de datos para crear modelos predictivos que sitúen un valor numérico o puntuación en la probabilidad de que ocurra un evento particular.

Cabe destacar resultados investigativos realizados desde diferentes perspectivas y la consideración de diferentes factores que enriquecen la presente investigación, por considerar la dimensión rendimiento académico e indicadores de carácter personal e institucional, así como estudios prospectivos, los cuales se referencian a continuación. En lo referente a los estudios sobre indicadores de carácter personal, se destacan los análisis de Paura y Arhipova (2014) sobre las causas de la tasa de deserción en el primer año de universidad, utilizando los datos reales del Programa de Estudios de Ingeniería en la Universidad de Agricultura de Letonia; se aplicó un modelo de riesgo proporcional de análisis de supervivencia y se encontraron entre las causantes de deserción: género de los estudiantes, calificaciones de la escuela secundaria con prioridad para estudiar en el programa y la fuente de financiamiento. 
Roso-Bas et al. (2016) destacan la perspectiva interaccionista ofrecida por Tinto (2006), en la que los aspectos de carácter institucional tienen una gran relevancia al explicar y administrar la retención de estudiantes. Sobre el indicador institucional periodo académico, Ameri et al. (2016) desarrollan un análisis marco de supervivencia para la predicción temprana de la deserción estudiantil, usando un Modelo de riesgos proporcionales de Cox y arriban a la conclusión de que los estudiantes más vulnerables son los de primer año, quienes corren el mayor riesgo de abandonar al inicio de sus estudios; por tanto, la identificación temprana de los estudiantes en riesgos es una tarea crucial que debe ser efectivamente dirigida.

Magen-Nagar et al. (2017) consideran variables personales para explicar el riesgo de abandono estudiantil; presentan como hallazgos que cuando existen relaciones interpersonales positivas, disminuye el riesgo de que los estudiantes abandonen. El número de estudiantes en la clase es otro indicador significativo en un estudio sobre los factores institucionales que influyen en el abandono y que ha sido señalado por Tinto (1975). Montmarquette et al. (2001) señalan que, en los cursos obligatorios de primer año, por lo general enseñado por un docente experimentado, afecta la probabilidad de persistencia de forma no lineal; con un conjunto de datos longitudinales, el análisis estadístico presentado por estos autores ofrece resultados interesantes sobre determinantes de abandonos universitarios, relacionados con un efecto del tamaño de las aulas en los cursos obligatorios del primer semestre y a detalles particulares del tipo de programa universitario. Diversos autores han desarrollado investigaciones con el objetivo de establecer modelos predictivos del abandono escolar. Bernardo, et al. (2015) mencionan los modelos de Castaño et al. (2004), Trevizán et al. (2009), Goldenhersh et al. (2011), Sánchez (2014), y en su investigación aplicaron la primera de las metodologías mencionadas, a fin de comprobar el valor predictivo de las variables: rendimiento académico previo, fecha de matriculación, rendimiento en primer curso de universidad y asistencia a clase; dichos resultados son consistentes con los obtenidos por diversos autores a lo largo del tiempo y en base a ellos se proponen acciones encaminadas a facilitar el diagnóstico respecto al problema del abandono y medidas encaminadas a su prevención.

Muchos han sido los esfuerzos en políticas pública para dar acceso masivo a las universidades; pero al mismo tiempo, la tasa de deserción ha llegado a constituirse en uno de los principales indicadores de eficiencia, dado el nivel tan elevado de deserción. Es por ello, que detectar las causas de la deserción universitaria y predecir ésta con mayor efectividad, permitirían mejorar la gestión universitaria en lo referente a la relación ingresopermanencia para que los estudiantes puedan alcanzar sus aspiraciones profesionales. La Universidad de los Andes (ULA) es una institución de Educación Superior (IES) venezolana, con más de 230 años formando profesionales en diversas áreas de conocimiento. Actualmente tiene_aproximadamente una población estudiantil de 60.000 estudiantes. Entre sus facultades se encuentran la Facultad de Ingeniería con las carreras de Ingeniería Civil , Ingeniería de Sistemas, Ingeniería Eléctrica , Ingeniería Mecánica e Ingeniería Química. La facultad de Ciencias Económica y Sociales dicta cuatro carreras: Economía, Administración de Empresas, Contaduría Pública y Estadística. Además, la facultad de Arquitectura y Diseño ofertan dos carreas: Arquitectura y Diseño Industrial.

La ULA, a pesar de ser una universidad con una trayectoria de largos años de experiencia, no se escapa del problema que enfrentan la mayoría de las IES del mundo sobre la deserción estudiantil y, por consiguiente, no es ajena a la necesidad de conocer y enfrentar el problema. El caso del abandono en las Matemáticas Básicas es de alta repercusión en la universidad, por lo que esta enfrenta la paradoja de aulas vacías, causadas por la deserción y al mismo tiempo estudiantes esperando cupos para poder cursarla. Herzog (2005) muestra que, en el caso de Estados Unidos, el haber aprobado un curso de Matemáticas de primer año, es uno de los factores que influye en la probabilidad de que un estudiante se matricule en el segundo año de estudios; asimismo, sostiene que la Matemática es un indicador de riesgo de abandono. De lo anterior, surge el interés de las autoridades de la institución objeto de estudio y de las direcciones de las Oficinas de Registro estudiantiles (ORE), de lograr una administración eficiente de los recursos e incrementar la retención de los estudiantes en las aulas de las Matemáticas Básicas (MB). Sobre estas bases, los autores se han propuesto estudiar, si las medidas de ofrecer cursos intensivos, cursos de libre escolaridad y la de regular el número de estudiantes por curso de Matemáticas, disminuyen la probabilidad de desertar; de igual manera conocer si las mujeres, por ser un sector vulnerable, tienen o no mayor probabilidad de desertar en dichos cursos. El objetivo general de la investigación es describir las probabilidades de deserción estudiantil en las Matemáticas Básicas de diferentes Programas Profesionales de la ULA.

La investigación se realiza en el período de 2011 a 2016 y asume un diseño de investigación cuantitativo. Se sigue la alternativa metodológica del estudio de caso único y el cumplimiento del objetivo propuesto fue posible a partir de la aplicación de diferentes métodos del nivel teórico, empírico y estadístico - matemático. Los autores reconocen la existencia de profusas investigaciones sobre la deserción y que uno de los principales consensos es considerarlo un fenómeno de múltiples causas y aristas, por tal motivo comprenden que ningún enfoque podrá abordar la totalidad del fenómeno. Por ello, la importancia de este trabajo reside en que no se ha trabajado como variables institucionales, para explicar la deserción, los cursos intensivos y los cursos de libre escolaridad. 


\section{METODOLOGÍA}

Se asume un diseño de investigación cuantitativo, al adoptar un enfoque que pretende descubrir nuevos conocimientos, explicarlos y predecirlos; se considera que el estudio del conocimiento de un momento dado conduce a la formulación de nuevas hipótesis o proposiciones, en las cuales se interrelacionan variables, cuya medición cuantitativa a través de procedimientos estadísticos, posibilitará comprobarlas o refutarlas en el proceso de investigación (Hernández et al., 2010). Se sigue la alternativa metodológica del estudio de caso, considerada como una investigación que, mediante los procesos cuantitativos, cualitativos y mixto, se analiza profundamente una unidad para responder al planteamiento del problema, probar una hipótesis o proposición y desarrollar teoría (Hernández, et al., 2010).

Teniendo en cuenta el tipo de datos recolectados, el estudio de caso asumido es de tipo cuantitativo, puesto que aplica el proceso cuantitativo al estudio de una unidad y se utilizan herramientas estandarizadas. A su vez, se considera no experimental de tipo transversal, que implica recolectar datos cuantitativos sobre una unidad en un momento único y no se manipula una intervención o tratamiento (Hernández et al., 2010). Atendiendo al objetivo de la estrategia de investigación, el estudio propuesto es de alcance descriptivo, porque su finalidad es analizar cómo ocurre el fenómeno estudiado dentro de su contexto real (Yin, 1994, citado por Hernández et al., 2010).

El tipo de diseño utilizado para el estudio de caso ha sido de un único caso, el cual se descompone en subunidades de análisis contenidas dentro del caso (Yin, 2009). La unidad de análisis es la ULA y las subunidades contenidas dentro del caso son: Facultad de Ingeniería, Facultad de Ciencias Económicas y Sociales y la Facultad de Arquitectura y Diseño. El proceso de investigación se realizó durante el periodo comprendido entre septiembre de 2011 a septiembre de 2016. Como propone Yin (2009) para este tipo de investigación, la estrategia de análisis que sigue consiste en: confiar en la teoría, organizar y efectuar el análisis sobre la base de las presuposiciones, reflexionar y evaluar explicaciones rivales y desarrollar cuidadosamente la descripción del caso y su contexto; en este particular la descripción se hace por subunidades del caso.

Para obtener la información y recolectar los datos necesarios en la presente investigación se ampliaron los siguientes métodos: i) Del nivel teórico: analítico-sintético: permitió establecer los fundamentos teóricometodológicos de la investigación, el diseño y el análisis de los resultados; inductivo-deductivo: confirmó las formulaciones teóricas en la realización de generalizaciones provenientes de los momentos empíricos para arribar a las generalizaciones y a conclusiones lógicas que afectan el fenómeno estudiado y sistémico estructural: contribuyó a determinar los componentes fundamentales del modelo de elección discreta (Logit) y sus interrelaciones. ii) Del nivel empírico: el análisis documental: se revisaron documentos oficiales de carácter interno como reglamentos e informes institucionales a partir de las categorías definidas, con el objetivo de valorar las necesidades de la Universidad de proyectarse hacia el trazado de políticas para responder a la deserción estudiantil. iii) Del nivel estadístico-matemático: un modelo de elección discreta (Logit) que permite determinar los factores que afectan o no la probabilidad de ocurrencia de un evento (Novales, 1993). El término logit procede de los "log add", que hace referencia a las probabilidades relativas de que una respuesta se encuentre en algunas de las categorías de la variable dependiente. Los resultados se obtienen empleando el SPSS en su versión 22 y los modelos logísticos tienen la forma:

$$
y_{1}=\beta_{0}+\beta_{1} x_{1}+\ldots+\beta_{i} x_{i}=E(y)=\frac{e^{x^{\prime} \beta}}{1+e^{x^{\prime} \beta}}=\frac{1}{1+e^{-x^{\prime} \beta}}=\pi_{i}
$$

Donde: $\pi$ corresponde a la probabilidad de ocurrencia del evento, $x$ al vector de variables explicativas, $\beta$ al vector de parámetros. Cuando $y_{i}$ es 1 la probabilidad es igual a $\pi_{i}$ y cuando es 0 , la probabilidad es $1-\pi_{i}$.

Variables: Siguiendo a Hinmel (2002) sobre los enfoques para el análisis de la deserción, se ha asumido el enfoque organizacional. Los criterios para la determinación de las variables de estudio están dados en: i) El interés de la ULA por las variables seleccionadas, con la finalidad de establecer políticas institucionales al respecto. ii) Dos de los investigadores eran directores de las ORE de la Universidad, por lo que tenían acceso a la información y posibilidades de responder a los intereses organizacionales.

Se ha considerado como variable dependiente la Deserción Estudiantil en las Matemáticas Básicas (DEMB) en las carreras que se dictan en las facultades de: Arquitectura y Diseño (FAD-ULA), Ciencias Económicas y Sociales (FACES-ULA) e Ingeniería (FI-ULA) y como variables explicativas o independientes: el Género del Estudiante (GE), el tipo de Periodo Académico (PA), el Tipo de curso Académico (TC) y el Número de Estudiantes (NE). En la Tabla 1 se muestra las variables categorizadas en formato binario y en la Figura 1 la frecuencia de ocurrencia DEMB, donde se destaca que el $41 \%$ de los estudiantes de la muestra desertó de 
la MB, valor que a primera vista es preocupante. Su distribución por facultad resalta un predominio de la no deserción en la mayoría de las facultades, excepto en FACES-ULA.

Tabla 1: Categorización de las variables

\begin{tabular}{|c|c|c|c|}
\hline \multicolumn{2}{|c|}{ Tipo } & Nombre & Descripción \\
\hline Dependiente & Institucional & $\begin{array}{l}\text { Deserción Estudiantil } \\
\text { en las Matemáticas } \\
\text { Básicas (DEMB) }\end{array}$ & $\begin{array}{l}\text { Un estudiante una vez matriculado en Matemáticas Básicas } \\
\text { (MB) la retira legalmente o la abandona. Vale } 1 \text { cuando el } \\
\text { estudiante deserta y } 0 \text { cuando no. }\end{array}$ \\
\hline \multirow{4}{*}{ Independiente } & Personal & $\begin{array}{l}\text { Género } \\
\text { (GE) }\end{array}$ & $\begin{array}{l}\text { Se refiere al género del estudiante, toma el valor } 1 \text { cuando } \\
\text { es femenino y } 0 \text { cuando es masculino. }\end{array}$ \\
\hline & \multirow{3}{*}{ Institucional } & $\begin{array}{l}\text { Tipo de Periodo } \\
\text { Académico (PA) }\end{array}$ & $\begin{array}{l}\text { Se refiere al régimen académico en el cual la materia se } \\
\text { cursó, toma el valor } 1 \text { cuando el régimen es regular ( } 16 \\
\text { semanas académicas) y } 0 \text { cuando el régimen es intensivo } \\
\text { ( } 6 \text { semanas académicas). }\end{array}$ \\
\hline & & $\begin{array}{l}\text { Tipo de Curso } \\
\text { Académico } \\
\text { (TC) }\end{array}$ & $\begin{array}{l}\text { Se refiere a la modalidad de enseñanza, toma el valor } 1 \\
\text { cuando es un curso especial por libre escolaridad ( } \sin \\
\text { clases presenciales) y } 0 \text { cuando es un curso regular. }\end{array}$ \\
\hline & & $\begin{array}{l}\text { Número de } \\
\text { estudiantes (NE) }\end{array}$ & $\begin{array}{l}\text { Se refiere al número total de estudiantes matriculados. } \\
\text { Toma valor } 1 \text { cuando se matriculan un total }>=\text { a } 50 \\
\text { estudiantes y } 0 \text { cuando es }>\text { a } 50 \text {. }\end{array}$ \\
\hline
\end{tabular}

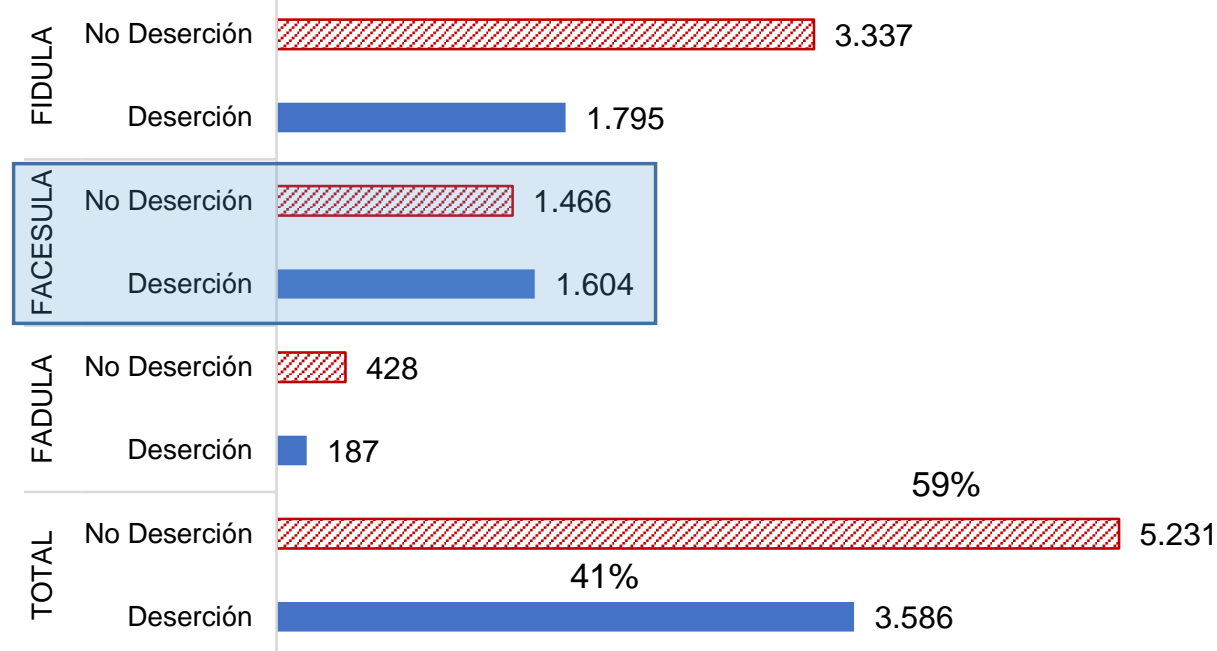

Fig. 1: Frecuencia de ocurrencia de la variable dependiente

Muestra: Siguiendo a Hernández et al. (2010), el procedimiento utilizado para la determinación de la muestra fue el muestreo por conveniencia y se siguieron criterios espaciales y funcionales. La investigación se realiza en la ULA, específicamente en los cursos de MB que se dictaron en las carreras de FAD-ULA, FACES-ULA y FI-ULA, durante el periodo septiembre 2011 y diciembre 2014.

Se consideran como atributos de criterio muestral: i) La localidad, dada la gran importancia que tiene la ULA en la formación de profesionales para el país y la región en donde se encuentra ubicada, la ciudad de Mérida. ii) La posibilidad institucional y capacidad para dar respuesta a los resultados de la investigación; contar con investigadores de larga experiencia laboral, la accesibilidad y proximidad de los datos para ellos. iii) Tres de los investigadores son docentes de la institución en las facultades estudiadas. Dos de ellos ejercieron funciones como directores de dos de las ORE de la institución. iv) las carreras antes mencionadas tienen como elemento común el dictado de las MB como asignatura nuclear de sus pensum de estudios.

De esta forma se seleccionó una muestra por conveniencia integrada por: tres facultades (FAD-ULA, FACESULA y FI-ULA), 10 carreras (Ingeniería Civil, Eléctrica, Geológica, Mecánica, Química, Sistema, Arquitectura, Economía, Administración de Empresas y Contaduría. Para un total de 8.817 matriculaciones en los cursos de MB durante el periodo 2011 -2014. Los datos registran el número de veces que se matricularon los estudiantes para cursar la asignatura durante el periodo en estudio, un estudiante pudo haberse matriculado una o más veces. En la Tabla 2 se presenta la distribución de la muestra: 
Tabla 2: Distribución de la muestra

\begin{tabular}{|c|c|c|c|c|c|c|c|c|c|}
\hline \multirow{2}{*}{\multicolumn{3}{|c|}{ Facultad/Carrera }} & \multicolumn{2}{|c|}{ Estudiantes } & \multirow{2}{*}{\multicolumn{3}{|c|}{ Facultad/Carrera }} & \multicolumn{2}{|c|}{ Estudiantes } \\
\hline & & & \multirow{2}{*}{$\begin{array}{c}\# \\
1.120\end{array}$} & \multirow{2}{*}{$\begin{array}{c}\% \\
12,7\end{array}$} & & & & & \\
\hline \multirow{6}{*}{$\begin{array}{l}\frac{\nwarrow}{5} \\
\frac{1}{\square}\end{array}$} & \multirow{6}{*}{$\frac{\widehat{\widetilde{m}}}{\text { in }}$} & Civil (CIC) & & & FAD-ULA & $(615)$ & Arquitectura (CA) & 615 & 7,0 \\
\hline & & Eléctrica (CIE) & 914 & 10,4 & \multirow{3}{*}{ FACES -ULA } & \multirow{3}{*}{$(3.070)$} & Economía (CE) & 854 & 9,7 \\
\hline & & Geológica (CIE) & 463 & 5,3 & & & Administración (CA) & 1.132 & 12,8 \\
\hline & & Mecánica (CIM) & 1.078 & 12,2 & & & Contaduría (CC) & 1.084 & 12,3 \\
\hline & & Química (CIQ) & 470 & 5,3 & \multirow{2}{*}{\multicolumn{3}{|c|}{ Total de la muestra }} & \multirow{2}{*}{\multicolumn{2}{|c|}{8.817}} \\
\hline & & Sistema (CIS) & $\begin{array}{r}1.08 \\
7\end{array}$ & 12,3 & & & & & \\
\hline
\end{tabular}

Especificación del modelo estimado: El modelo estimado para cada una de las facultades (FACES-ULA, FADULA y FI-DULA) es el siguiente. En FAD-ULA no se dan cursos por libre escolaridad.

$$
\Pi_{D E M B}=\beta_{0}+\beta_{1} G E+\beta_{2} P A+\beta_{3} T C+\beta_{4} N E
$$

En esta ecuación, $\Pi_{D E M B}$ corresponde a la probabilidad de desertar de las MB; $x_{1}$ al Género del Estudiante (GE); $x_{2}$ al Tipo de Periodo Académico (PA); $x_{3}$ al Tipo de Curso (TC) y x4 al Número de Estudiantes (NE).

\section{RESULTADOS}

Análisis descriptivo y correlaciones: La Tabla 3 destaca un predominio de estudiantes del género masculino, cursos con más de cincuenta estudiantes y asignatura cursada en cursos regulares. Para el caso de tipo de periodo académico la muestra es homogénea.

Tabla 3: Categorización, frecuencia y distribución de las variables independientes

\begin{tabular}{|c|c|c|c|c|c|c|c|c|c|c|}
\hline \multirow{2}{*}{ Variable } & \multirow{2}{*}{\multicolumn{2}{|c|}{ Categorización }} & \multicolumn{2}{|c|}{ GLOBAL } & \multicolumn{2}{|c|}{ FACES-ULA } & \multicolumn{2}{|c|}{ FAD-ULA } & \multicolumn{2}{|c|}{ FI-ULA } \\
\hline & & & $\#$ & $\%$ & \# & $\%$ & \# & $\%$ & \# & $\%$ \\
\hline \multirow{2}{*}{ Género del Estudiante (GE) } & Masculino & 0 & 5163 & 59 & 1242 & 40 & 291 & 47 & 3630 & 71 \\
\hline & Femenino & 1 & 3654 & 41 & 1828 & 60 & 324 & 53 & 1502 & 29 \\
\hline \multirow{2}{*}{$\begin{array}{l}\text { Tipo de Periodo Académico } \\
\text { (PA) }\end{array}$} & Intensivo & 0 & 4337 & 49 & 1299 & 42 & 241 & 39 & 2797 & 55 \\
\hline & Regular & 1 & 4480 & 51 & 1771 & 58 & 374 & 61 & 2335 & 45 \\
\hline \multirow{2}{*}{ Tipo de Curso (TC) } & Regular & 0 & 8073 & 92 & 2719 & 89 & ---- & --- & 4739 & 92 \\
\hline & Especial & 1 & 744 & 8 & 351 & 11 & ---- & --- & 393 & 8 \\
\hline \multirow{2}{*}{$\begin{array}{l}\text { Número de Estudiantes por } \\
\text { Curso (NE) }\end{array}$} & Mayor a 50 & 0 & 6677 & 76 & 2394 & 78 & 334 & 54 & 3949 & 77 \\
\hline & Menor Igual a 50 & 1 & 2140 & 24 & 676 & 22 & 281 & 46 & 1183 & 23 \\
\hline
\end{tabular}

Las correlaciones entre DEMB y las variables seleccionadas en los modelos teóricos se presentan en la figura 2. Se aprecia que las variables que presentan relación positiva con DEMB son: el TC (25\%) y el PA (10\%). En cuanto a NE su relación con la deserción es negativa y GE presenta muy poca correlación.

Ajuste global del modelo: En la tabla 4 se muestran los indicadores de ajuste del modelo y se destaca que: la proporción de la variabilidad de DEMB explicada por el modelo oscila entre $1,7 \%$ y $10,1 \%$, lo que significa, especialmente para FAD-ULA, que sigue existiendo un porcentaje importante de influencia sobre el hecho de desertar en las MB que no depende de las variables analizadas en este modelo. Con estos valores sería arriesgado proponer un modelo de predicción como conclusión de este estudio. La prueba de la significancia global nos permite rechazar la hipótesis de que los coeficientes de todas las variables explicativas son cero; por lo tanto, la probabilidad de ocurrencia de los valores que representan esta calificación varía para alguna de las combinaciones de valores de las variables independientes. Para FAD-ULA el modelo es significativo al 6,5\%. 


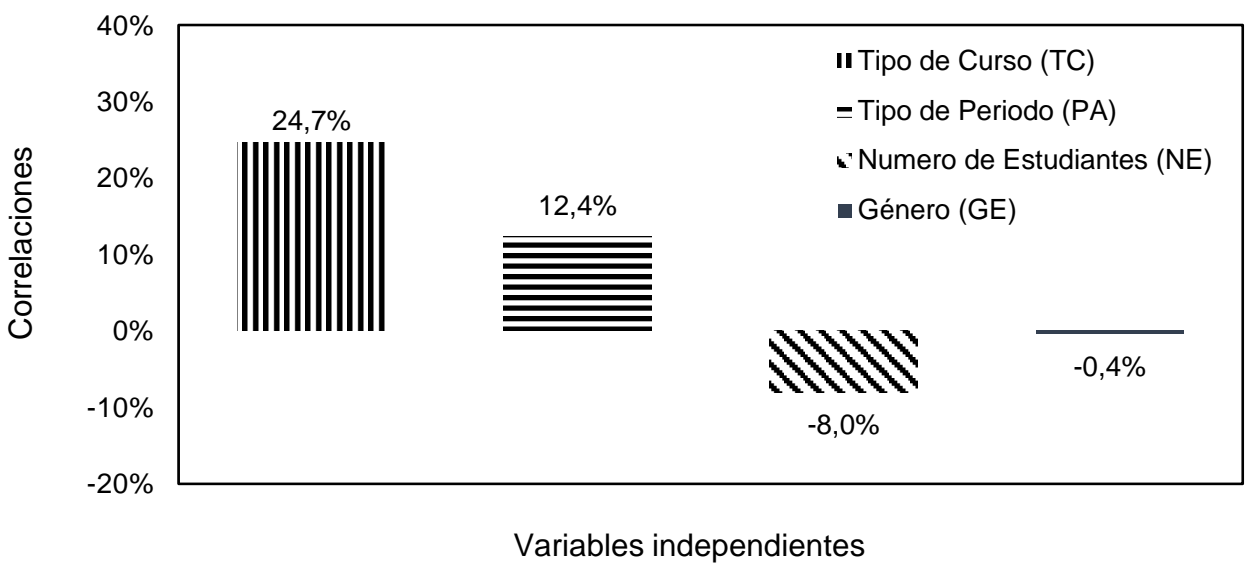

Fig. 2: Correlaciones de la variable dependiente vs las independientes

El modelo tiene una capacidad de clasificar correctamente al 59\%, 70\% y 69\% (FACES-ULA, FAD-ULA y FIULA respectivamente) de los casos analizados. Para el caso de FACES-ULA clasifica mejor a la deserción y en FAD-ULA Y FI-ULA a la no deserción. Adicionalmente, los valores del test Chi Cuadrado de la prueba de Hosmer Lemeshow nos indican que no hay motivos para pensar que los resultados estimados sean diferentes de los observados y que el modelo puede considerarse aceptable.

Tabla 4: Ajuste del modelo

\begin{tabular}{|l|c|c|c|c|c|c|}
\hline \multirow{2}{*}{ Facultad } & \multirow{2}{*}{$R^{2}$ Logit } & \multicolumn{2}{|c|}{ Test Global $\left(X^{2}\right)$} & \multirow{2}{*}{ \%Correcto } & \multicolumn{2}{|c|}{ Test Hosmer Lemeshow } \\
\cline { 3 - 4 } \cline { 6 - 7 } & & Estadístico & $p$-value & & Estadístico & $p$-value \\
\hline FACES-ULA & 0,098 & 234,289 & 0,000 & 59,1 & 10,771 & 0,096 \\
\hline FAD-ULA & 0,017 & 7,244 & 0,065 & 69,6 & 7,485 & 0,187 \\
\hline FI-ULA & 0,101 & 391,815 & 0,000 & 69,1 & 6,999 & 0,321 \\
\hline
\end{tabular}

Análisis de resultados: Tal como se muestra en la tabla 5, las variables que resultan relevantes en la decisión de desertar o no son PA y TC, para FACES-ULA y FI-ULA, GE en las tres facultades, aunque hay que recordar que su correlación con la variable dependiente fue muy baja y $N E$ en FI-ULA.

Tabla 5: Resultados del modelo de regresión logística

\begin{tabular}{|l|l|c|c|c|c|}
\hline Facultad & \multicolumn{1}{|c|}{ Variables Independientes } & $\beta$ & Estadístico Wald & $p$-value & Odds Ratio \\
\hline \multirow{4}{*}{$\begin{array}{l}\text { FACES- } \\
\text { ULA }\end{array}$} & Constante & $-0,202$ & 6,773 & 0,009 & 0,817 \\
\cline { 2 - 6 } & Género del Estudiante (GE) & $-0,236$ & 9,573 & 0,002 & 0,789 \\
\cline { 2 - 6 } & Tipo de Periodo Académico (PA) & 0,451 & 34,861 & 0,000 & 1,571 \\
\cline { 2 - 6 } & Tipo de Curso (TC) & 1,785 & 128,449 & 0,000 & 5,958 \\
\cline { 2 - 6 } & Número de Estudiantes (NE) & 0,035 & 0,148 & 0,701 & 1,035 \\
\hline \multirow{4}{*}{ FAD-ULA } & Constante & $-0,806$ & 8,044 & 0,005 & 0,447 \\
\cline { 2 - 6 } & Género del Estudiante (GE) & $-0,412$ & 5,449 & 0,020 & 0,662 \\
\cline { 2 - 6 } & Tipo de Periodo Académico (PA) & 0,265 & 1,043 & 0,307 & 1,303 \\
\cline { 2 - 6 } & Número de Estudiantes (NE) & 0,050 & 0,039 & 0,843 & 1,051 \\
\hline \multirow{5}{*}{ FI-ULA } & Constante & $-0,863$ & 303,942 & 0,000 & 0,422 \\
\cline { 2 - 6 } & Género del Estudiante (GE) & $-0,200$ & 8,649 & 0,003 & 0,819 \\
\cline { 2 - 6 } & Tipo de Periodo Académico (PA) & 0,475 & 60,009 & 0,000 & 1,608 \\
\cline { 2 - 6 } & Tipo de Curso (TC) & 1,878 & 225,331 & 0,000 & 6,541 \\
\cline { 2 - 6 } & Número de Estudiantes (NE) & $-0,330$ & 19,226 & 0,000 & 0,719 \\
\hline
\end{tabular}

Las ecuaciones estimadas son las siguientes:

$$
\begin{aligned}
& \Pi_{\text {DEMB-FACES-ULA }}=-0,202-0,236 \mathrm{GE}+0,451 \mathrm{PA}+1,785 \mathrm{TC}+0,035 \mathrm{NE} \\
& \Pi_{\text {DEMB-FAD-ULA }}=-0,806-0,412 \mathrm{GE}+0,265 \mathrm{PA}+0,050 \mathrm{NE} \\
& \Pi_{\text {DEMB-FI-ULA }}=-0,863-0,200 \mathrm{GE}+0,475 \mathrm{PA}+1,878 \mathrm{TC}-0,330 \mathrm{NE}
\end{aligned}
$$


Los valores Odds indican: en cuanto a la variable $P A$ que aquellos estudiantes que cursan la asignatura durante un semestre regular tienen mayor probabilidad de desertar que aquellos que lo cursan en un semestre intensivo. En FACES-ULA tienen 1,571 más probabilidad de desertar si la cursan en un semestre regular que un semestre corto y en FI-ULA 1,698. En cuanto a la variable $T C$ muestran que aquellos estudiantes que se matriculan en un curso especial (libre escolaridad) tienen mayor probabilidad de desertar que aquellos que la cursan en los cursos regulares. En FACES-ULA tienen 5,958 más probabilidad de desertar si cursan la asignatura en un curso de libre escolaridad y en FI-ULA 6,541. En cuanto GE revelan que las estudiantes tienen menos probabilidad de desertar que los hombres. En FACES-ULA las estudiantes tienen 1,23 (1/0,789) veces menos probabilidad de desertar que sus pares hombres, en FAD-ULA 1,51 (1/0,662) y en FI-ULA 1,22 $(1 / 0,819)$. En cuanto NE indica que en FI-ULA los estudiantes que cursan la asignatura en curso con menos de cincuenta estudiantes matriculados tienen $1,23(1 / 0,789)$ veces menos probabilidad de desertar de quienes las cursan en cursos con más de cincuenta estudiantes matriculados. En las otras facultades esta variable no resultó estadísticamente significativa.

De la muestra analizada se destaca que una estudiante mujer que curse la asignatura en un curso especial con más de cincuenta estudiantes matriculados y en un periodo académico regular tiene mayor probabilidad de desertar que los estudiantes masculinos que cursan la asignatura en un semestre corto, en un curso regular y con menos de cincuenta estudiantes matriculados. Las variables $P A$ y $T C$ tienen un efecto positivo sobre la deserción en las MB, mientras que GE un efecto negativo, y NE un efecto negativo para el caso de FI-ULA y positivo para las otras dos facultades. Muñoz-Camacho et al. (2016), utilizando la misma muestra de este trabajo, pero incluyendo como variables explicativas la carrera cursada por los estudiantes y el docente que dictó la asignatura, encontraron que ambas variables son elementos condicionantes a la decisión de abandonar el curso de Matemáticas.

En lo referente a GE, estudios como los de Soria-Barreto (2014), Ibarra (2010) y Barahona (2014) encontraron que el género muestra una relación positiva con el éxito de los estudiantes, siendo las mujeres quienes obtienen una mayor tasa de éxito y por lo tanto menor probabilidad de desertar, visto de manera general en todas las asignaturas del currículo; reflejan el hecho de que una estudiante mujer asume un mayor compromiso en los estudios, lo que se ve reflejado en un mejor rendimiento académico. Sánchez y Zuñiga (2012) sostienen en su estudio que los hombres desertan más que las mujeres, aunque para ambos géneros ha habido un incremento similar en la deserción.

Sin embargo, específicamente para las Matemáticas, los resultados investigativos muestran otra situación que guarda relación con lo obtenido en el presente trabajo. En estudios de Doyle y Voyer (2015) se sugiere que los estereotipos que existen afectan negativamente en la previsión del rendimiento de las mujeres en las Matemáticas; los hombres no se ven afectados por la amenaza o el estímulo y, por tal motivo, las manipulaciones de amenaza o estímulo afectan a las mujeres porque estas son creíbles a la luz del estereotipo. Franceschini et al. (2014) encontraron que los estereotipos implícitos de género en Matemáticas actúan como una variable crítica para determinar la autoeficacia y el rendimiento de las mujeres. Por su parte Henríquez (2016) encontró que la variable sexo incide en la probabilidad de aprobar la prueba de habilidades de Matemática, de tal forma que un estudiante hombre tiene 1.451 veces más probabilidad de aprobar que si es mujer; se reafirma entonces que el género masculino posee en promedio mejores rendimientos.

Sobre el NE existen numerosos estudios con posiciones encontradas. Monks y Schmidt (2011), Yusuf et al. (2016) encontraron que el tamaño de la clase afecta negativamente a los estudiantes de manera significativa en: las evaluaciones, las actitudes generales, de aprendizaje y de comportamiento, la puntualidad, la motivación y la participación, por lo que señalan la necesidad de ponerle especial atención en cada entorno educativo. Sin embargo, existen otros resultados que demuestran lo contrario, como el de Shen y Konstantopoulos (2017) quienes expresan que los resultados obtenidos no muestran efecto negativo; también existen estudios que muestran mejoras modestas en el rendimiento o que este efecto negativo es significativamente mayor para estudiantes de baja capacidad e insignificante para los de alta capacidad, como las investigaciones de Jepsen y Rivkin (2009) y De Paola et al. (2013). A pesar de las investigaciones realizadas sobre el tamaño de la clase, esta relación aún se desconoce.

Para futuros estudios y con fines de mejorar el modelo, se pueden incluir otras variables dependientes de tipo institucional, tales como: ayudas económicas, acompañamiento académico, flexibilidad curricular, etc. Y no menos interesante sería considerar variables socioeconómicas, rendimiento estudiantil, ingreso a universidad, entre otras. Se recomienda a la Universidad de Los Andes (ULA), realizar acciones para fomentar la retención y disminuir la deserción de los estudiantes en las asignaturas de Matemáticas, a la luz de los resultados obtenidos. Estas acciones deben diseñarse a partir de la aplicación del método de Análisis Multivalente a través de un modelo Logit, por ser el método más adecuado para realizar el análisis de la probabilidad de deserción o no en Matemática Básica. 


\section{CONCLUSIONES}

1.- En el ámbito latinoamericano, todavía son escasos los trabajos sobre la deserción estudiantil en la Educación Superior con una orientación predictiva. El método más adecuado para realizar el análisis de la probabilidad de deserción o no en Matemática Básica es el Análisis Multivalente a través de un modelo Logi.t

2.- Aquellos estudiantes que cursan la asignatura durante un semestre regular tienen mayor probabilidad de desertar que aquellos que lo cursan en un semestre intensivo; en la Facultad de Ciencias Económicas y Sociales (FACESULA) tienen 1,571 más probabilidad de desertar, si la cursan en un semestre regular que un semestre corto y en la Facultad de Ingeniería (FI-ULA) 1,698. Por su parte, aquellos estudiantes que se matriculan en un curso especial (libre escolaridad) tienen mayor probabilidad de desertar que aquellos que la cursan en los cursos regulares; en FACES-ULA tienen 5,958 más probabilidad de desertar, si cursan la asignatura en un curso de libre escolaridad y en FI-ULA 6,541.

3.- Las estudiantes tienen menos probabilidad de desertar que los hombres. En FACES-ULA las estudiantes tienen 1,23 $(1 / 0,789)$ veces menos probabilidad de desertar que sus pares hombres, en la Facultad de Arquitectura y Diseño (FAD-ULA) 1,51 (1/0,662) y en FI-ULA 1,22 (1/0,819).

4.- Los estudiantes que cursan la asignatura en curso con menos de cincuenta estudiantes matriculados en el curso tienen $1,23(1 / 0,789)$ veces menos probabilidad de desertar de quienes las cursan en cursos con más de cincuenta estudiantes matriculados. En las otras facultades esta variable no resultó estadísticamente significativa.

5.- De la muestra analizada se destaca que una estudiante mujer que curse la asignatura en un curso especial con más de cincuenta estudiantes matriculados y en un periodo académico regular tiene mayor probabilidad de desertar que los estudiantes masculinos que cursan la asignatura en un semestre corto, en un curso regular y con menos de cincuenta estudiantes matriculados. A su vez, es significativo que las variables Tipo de Periodo Académico (PA) y Tipo de Curso Académico (TC) tienen un efecto positivo sobre la deserción en las Matemáticas Básicas; mientras que el Género (GE), un efecto negativo y el Número de Estudiantes por curso (NE) tienen un efecto negativo para el caso de FI-ULA y positivo para las otras dos facultades

\section{AGRADECIMIENTOS}

Los autores agradecen a la Universidad de Los Andes, Mérida, Venezuela, por permitir el uso de sus datos.

\section{REFERENCIAS}

Ameri, S., M. Fard, R. Chinna y C. Reddy, Survival Analysis Based Framework for Early Prediction of Student Dropouts, in Proceedings of the 25th ACM International on Conference on Information and Knowledge Management, 903-912, ACM, Indianapolis, Indiana, USA, 24 a 28 de Octubre (2016)

Barahona P., Factores Determinantes del Rendimiento Académico de los Estudiantes de la Universidad de Atacama, doi: 10.4067/S0718-07052014000100002, Estudios Pedagógicos, 40 (1), 25-39 (2014)

Bernardo, A., R. Cerezo y otros cuatro autores, Predicción del Abandono Universitario: Variables Explicativas y Medidas de Prevención, doi: 10.12795/revistafuentes.2015.i16.03, Revista Fuentes, 63-84 (2016)

De Paola M., M. Ponzo y V. Scoppa, Class Size Effects on Student Achievement: Heterogeneity Across Abilities and Fields, doi: 10.1080/09645292.2010.511811, Education Economics, 21(2), 135-153 (2013)

Doyle, R., y D. Voyer, Stereotype Manipulation Effects on Math and Spatial Test Performance: A MetaAnalysis, doi:10.1016/j.lindif.2015.12.018, Learning and Individual Differences, 43, 103-116 (2016)

Franceschini, G., S. Galli, F. Chiesi y C. Primi, Implicit Gender-Math Stereotype and Women's Susceptibility to Stereotype Threat and Stereotype Lift, doi:10.1016/j.lindif.2014.03.020, Learning and Individual Differences, 32, 273-277(2014)

Hammond, C., D. Linto, J. Smink y S. Drew, Dropout Risk Factors and Exemplary Programs: A Technical Report. 1-268, National Dropout Prevention Center at Clemson University and Communities in Schools, EE.UU. (2007)

Henríquez, N. y D. Escobar, Construcción de un Modelo de Alerta Temprana para la Detección de Estudiantes en Riesgo de Deserción de la Universidad Metropolitana de Ciencias de la Educación, Revista Mexicana de Investigación Educativa, 21(71), (2016)

Hernández, R., C. Fernández y P. Batista, Metodología de la Investigación, 5ª Ed., 52-65, Mcgraw-Hill Interamericana, México D.F., México (2010)

Herzog, S., Measuring Determinants of Student Return vs. Dropout/Stopout vs.Transfer: A First-to-Second Year Analysis of Freshmen, Research in Higher Education, 883-928 (2005) 
Himmel, E., Modelos de Análisis de la Deserción Estudiantil en la Educación Superior, Revista Calidad de la Educación, Consejo Superior de Educación, Ministerio de Educación, Chile, 17, 91-108 (2002)

Ibarra, C. y J. Michalus, Análisis del Rendimiento Académico Mediante un Modelo Logit, Revista Industrial, 9(2), 47-56 (2010) Jepsen, C. y S. Rivkin, Class Size Reduction and Student Achievement: The Potential Tradeoff Between Teacher Quality and Class Size, Journal of Human Resources, 44, 223-250 (2009)

Magen-Nagar, N. y H. Shachar, Quality of Teaching and Dropout Risk: A Multi-Level Analysis, doi:10.1080/10824669.2016.1242069, Journal of Education for Students Placed at Risk (JESPAR), 22 (1), 9-24 (2017)

Mera, C., Pensamiento Prospectivo: Visión Sistémica de la Construcción del Futuro, Revista Análisis, ISSN: 0120-845446, (84), $89-10$ (2014)

Miranda, M. y J. Guzmán, Análisis de la Deserción de Estudiantes Universitarios usando Técnicas de Minería de Datos, doi: 0.4067/S0718-50062017000300007, Formación Universitaria, 10(3), 61-68 (2017)

Montmarquette, C., S. Mahseredjian y R. Houle, The Determinants of University Dropouts: a Bivariate Probability Model with Sample Selection, Economics of Education Review 20, 475-484 (2001)

Monks, J. y R. Munning, The Impact of Class Size on Outcomes in Higher Education, doi: 10.2202/1935-1682.2803, The B.E. Journal of Economic Analysis and Policy,11(1), 1-17 (2011)

Muñoz-Camacho, S., M. Muñoz, C. Muñoz y T. Gallardo, Abandono Etudiantil en la Educación Superior: Caso de Estudio sobre las Matemáticas Básicas como Factor Asociado, VI CLABES, Sexta Conferencia Latinoamericana sobre el abandono en la educación superior, 9-11, (2016)

Narváez, A., El Rendimiento Académico una Cuestión de Estudio, ARA Editores, Perú (2001)

Novales, A., Econometría, 2ª Ed., 1-676, Editorial McGraw-Hill (1993)

Ortega, F., El método Delphi, Prospectiva en Ciencias Sociales a través del Análisis de un Caso Práctico, Revista EAN, ISSN: 0120-8160, 64, 31-54 (2008)

Paura L., I. Arhipova, Cause Analysis of Students' Dropout Rate in Higher Education Study Program, Procedia - Social and Behavioral Sciences, 109, 1282-1286 (2014)

Roso-Bas, F., A. Pades, A. y E. García-Buades, Emotional Variables, Dropout and Academic Perfomance in Spanish Nursing Students, doi:10.1016/j.nedt.2015.11.021, Nurse Education Today, 37, 53-58 (2016)

Sánchez, F. y J. Márquez, La Deserción en la Educación Superior en Colombia durante la Primera Década del Siglo XXI. ¿Por qué ha aumentado tanto?, Documentos CEDE, Bogotá, Universidad de los Andes-CEDE (2012)

Shen, T., y S. Konstantopoulos, Class Size Effects on Reading Achievement in Europe: Evidence from Pirls, doi: 10.1016/j.stueduc.2017.04.001, Studies Educational Evaluation, 53, 98-114 (2017)

Soria-Barreto K. y S. Zúñiga-Jara, Aspectos Determinantes del Éxito Académico de Estudiantes Universitarios, doi: 10.4067/S0718-50062014000500006, Formación Universitaria, 7(5), 41-50 (2014)

Tinto, V., Dropouts from Higher Education: a Theoretical Synthesis of Recent Research. Review of Education Research 45, 89-125 (1975)

Yin, R., Case study research: Design and Methods, 4th Ed., Thousand Oaks, CA, SAGE (2009)

Yusuf, T., C. Onifade y O. Bello, Impact of Class Size on Learning, Behavioral and General Attitudes of Students in Secondary Schools in Abeokuta, Ogun State Nigeria. Journal of Research Initiatives, 2(1), 1-18 (2016) 